\title{
Enseignement supérieur, capital humain et croissance économique: Une approche par l'analyse causale
}

\author{
Drissa Touré, \\ Unisersité Felix Houphouet-Boigny, Abidjan-Cocody, \\ UFR Sciences de l'Homme et de la Société (SHS), \\ Institut des Sciences Anthropologiques de Développement, Côte d'Ivoire
}

Doi:10.19044/esj.2020.v16n16p255 URL:http://dx.doi.org/10.19044/esj.2020.v16n16p255

\section{Résumé}

La contribution de l'enseignement supérieur à la croissance économique dans les pays en voie de développement est très discutée. Le présent travail s'est intéressé au débat en analysant l'impact de cet ordre d'enseignement sur la croissance économique de la Côte d'Ivoire entre 1970 et 2016. Le stock de capital humain de niveau supérieur y est approché par la proportion des travailleurs ayant atteint au moins l'enseignement supérieur et la variable économique est représentée par le produit par travailleur. En utilisant la méthode d'estimation des variables autorégressives à retards échelonnés (ARDL), il résulte que l'enseignement supérieur ivoirien affecte négativement et significativement la croissance économique aussi bien à court terme qu'à long terme. La relation de long terme, confirmée par le test de cointégration aux bornes de Pesaran et al. (2001), montre une certaine sensibilité aux crises socio-politiques, les coefficients étant affectés durant la période de crise puis la constante pendant la période de sortie de crise. Certes, le test de Toda et Yamamoto (1995) révèle une causalité réciproque entre le stock de capital humain de niveau supérieur et le niveau de produit par travailleur. Mais, le signe négatif de leur coefficient et l'intensité causale en faveur de l'enseignement supérieur indiquent que celui-ci a un effet prohibitif sur l'accroissement de la richesse du pays. Il apparait nécessaire, pour inverser cet effet négatif, d'améliorer la qualité de la formation des étudiants, de lutter contre le chômage des diplômés et promouvoir la bonne gouvernance.

Mots clés : Enseignement supérieur, croissance économique, causalité, modèle ARDL 


\title{
A Causal Analysis Approach to Explain the Link Between Higher Education and Economic Growth
}

\author{
Drissa Touré, \\ Unisersité Felix Houphouet-Boigny, Abidjan-Cocody, \\ UFR Sciences de l'Homme et de la Société (SHS), \\ Institut des Sciences Anthropologiques de Développement, Côte d'Ivoire
}

\begin{abstract}
The contribution of higher education to economic growth in developing countries is highly debated. The present work is concerned with the debate by analyzing the impact of this level of education on the economic growth of Côte d'Ivoire between 1970 and 2016. The stock of higher-level human capital is approached by the proportion of workers who have attained at least higher education and the economic variable is represented by the product per worker. By using the method of estimating autoregressive distributed lag (ARDL), it follows that Ivorian higher education negatively and significantly affects economic growth in both the short and long terms. The long-term relationship, confirmed by the cointegration test at Pesaran et al. (2001), shows a certain sensitivity to socio-political crises, the coefficients being affected during the crisis period and then the constant during the exit period of crisis. Indeed, the test of Toda and Yamamoto (1995) reveals a reciprocal causality between the stock of higher-level human capital and the level of product per worker. However, the negative sign of their coefficient and the causal intensity in favor of higher education indicate that it has a prohibitive effect on the growth of the country's wealth. To reverse this negative effect, it seems necessary to improve the quality of student training, to combat unemployment among graduates and to promote good governance.
\end{abstract}

Keywords: Higher education, economic growth, causality, ARDL model

\section{Introduction}

Pour la théorie économique, il existe une relation entre le capital humain produit de l'éducation, la formation et la croissance économique (Boccanfuso et al, 2009). Cette relation jouerait principalement dans le sens de l'éducation en tant que cause ou facteur de croissance économique. De nombreux travaux empiriques ont testé le lien direct entre ces deux variables et ont attesté de l'impact positif de l'éducation sur la croissance économique 
des pays (Ali et al., 2018 ; Kotaskova et al., 2018 ; Nowak et Dahal, 2016). Certains chercheurs soutiennent que cet impact s'améliore considérablement et devient plus évident lorsqu'on prend en compte la dimension qualité du système éducatif dans les régressions (Hanushek et Woessmann, 2016 ; Okocha, 2015).

Les réserves émises par d'autres auteurs notamment Gurgand (2000) peuvent désormais être levées selon Ali et al. (2018). En effet, après avoir relevé les contradictions de certains travaux empiriques sur le rôle direct de l'éducation dans la croissance économique, Gurgand (2000) a posé le problème de spécification dans les modèles de croissance c'est-à-dire la question du lien fonctionnel direct entre éducation et le niveau de produit. Il note que s'il existe des travaux qui font apparaitre un lien positif, il y en a d'autres, comme Benhabib et Spiegel (1994) ou plus tard Prichett (2001), qui affichent un lien négatif. Même quand le lien est positif, sa significativité ne résiste pas à la rigueur méthodologique. Ali et al. (op. cit.) estiment que les résultats négatifs ou non significatifs constatés dans des études antérieures seraient dus à l'omission d'une variable exogène importante. Pour eux, cette variable, c'est « les capabilités sociales ». En l'intégrant dans leur modèle, ils obtiennent un effet positif et significatif.

Par ailleurs, l'analyse de la relation indirecte entre les deux variables atteste aussi que la formation du capital humain favorise la croissance économique. Sow (2013) l'a montré avec son étude sur le cas du Sénégal. L'auteur trouve que l'éducation impacte positivement la croissance du produit global du Sénégal à travers son effet positif sur la productivité des grandes entreprises du pays.

En somme, on retient que l'éducation est susceptible d'influer positivement et significativement la croissance économique. On en déduit que les pays ont intérêt à investir dans l'éducation de leurs populations et à améliorer la qualité de leurs systèmes éducatifs afin de favoriser la croissance économique et le développement.

Cependant, si l'éducation est importante pour la croissance économique et le développement des nations, son financement pose problème. En effet, l'éducation est coûteuse, et il existe d'autres secteurs aussi prioritaires face à des ressources limitées (la santé par exemple qui est aussi une composante du capital humain). Alors, faut-il assurer l'éducation de tous, et jusqu'où ? Cela conduit à faire un choix entre une éducation de masse et une éducation sélective et de qualité. Il s'agit aussi d'opérer un choix entre les différents niveaux du système éducatif : primaire, secondaire et supérieur. La réponse aurait été immédiate si on connaissait les niveaux d'éducation qui influencent le plus la croissance économique des pays. A ce sujet, il existe une divergence réelle entre les spécialistes. 
Pour certains, l'éducation primaire a plus d'impact sur la croissance économique et mérite d'être privilégiée (Keller, 2006 ; Colclough, 1982). Pour d'autres, les niveaux secondaire et supérieur présentent plus d'effets positifs et significatifs pour l'accroissement du produit global (Kocourek et Nedomlelova, 2018 ; Okocha, 2015). Malgré ces contradictions, il est à remarquer que le choix des politiques semble déjà fait. La plupart des gouvernements et les institutions internationales de développement incitent à une scolarisation primaire universelle au point que le niveau d'enseignement supérieur apparait moins dans les priorités de financement (Bloom et al, 2014). Car, en plus de ses effets positifs sur la croissance des pays en développement, l'éducation primaire est considérée comme un droit (Pritchett, 2001).

Toutefois, McNeil et Silim (2012) estiment que c'est l'enseignement supérieur qui fournit les travailleurs qualifiés, crée les conditions de l'innovation et apporte les plus grands bénéfices. Cet avis est certes partagé par Aghion et Cohen (2004) mais ils estiment qu'il y a lieu de nuancer. Pour ces auteurs, l'enseignement supérieur est plus productif pour les pays proches de la frontière technologique c'est-à-dire les pays développés tandis que l'enseignement primaire conviendrait mieux aux pays en développement, et le niveau secondaire de l'éducation serait plus adéquat pour les pays intermédiaires. Cette position est partagée par Vandenbussche et al. (2006) qui soutiennent que l'enseignement supérieur joue un rôle important pour la croissance des économies des pays développés alors qu'il a un impact moindre voire négatif pour les autres pays.

Ces analyses suscitent le débat sur la place de l'enseignement supérieur dans la croissance économique des pays en développement. La présente étude se veut une participation à ce débat en analysant la contribution de l'enseignement supérieur à la croissance économique de la Côte d'Ivoire, pays en voie de développement caractérisé par une croissance forte de son économie.

\section{Methodologie}

\section{II.1. Spécification du modèle de base}

On retient comme cadre théorique le modèle de base de Solow augmenté du capital humain, dissociant le capital humain du facteur travail tel que effectué par Mankiw et al. (1992). Ce modèle, à l'origine, est une fonction de production de type Cobb-Douglas qui s'écrit $Y=f(A, K, H)$ ou bien $Y=$ $A f(K, H)$ en considérant la neutralité du progrès technologique au sens de Hicks (Jeon, 2007). Dans les deux cas, la fonction explicite donne :

$$
Y=A K^{\alpha} H^{1-\alpha} \quad \text { (1) où : }
$$

Y est le produit ; A est le facteur de productivité totale ; K est le stock de capital physique et $\mathrm{H}$ est le stock de capital humain. 
En décomposant $\mathrm{H}$ en h, le niveau moyen de capital humain par travailleur et en L, la quantité de facteur travail, on obtient la nouvelle forme de cette fonction qui est :

$$
Y=A K^{\alpha}(h L)^{1-\alpha}
$$

En divisant chaque membre de l'égalité par $L$ on obtient successivement les équations ci-dessous:

$\frac{Y}{L}=\frac{A K^{\alpha}(h L)^{1-\alpha}}{L}$
$\frac{Y}{L}=A\left(\frac{K}{L}\right)^{\alpha}(h L)^{1-\alpha} L^{\alpha-1}$
$\frac{Y}{L}=A\left(\frac{K}{L}\right)^{\alpha} h^{1-\alpha} L^{1-\alpha} L^{\alpha-1}$
$\frac{Y}{L}=A\left(\frac{K}{L}\right)^{\alpha} h^{1-\alpha}$

En posant $y=\frac{Y}{L} ; k=\frac{K}{L}$, on obtient : $y=A k^{\alpha} h^{1-\alpha}$

avec $y$ : le niveau de produit par unité de travail ; $k$ : le stock de capital physique par unité de travail ; $h$ : le stock moyen de capital humain.

Considéré par Solow (1956) comme facteur technologique exogène, A est considéré dans les modèles de croissance comme les effets des changements technologiques, des institutions et de tout autre facteur de productivité intangible (Kohli, 2015). A cet égard, il est une fonction de plusieurs variables. Ainsi, estimant qu'il est le résultat d'un système financier et d'un cadre institutionnel développés, Keho (2012) pose pour une période $\mathrm{t}$ donnée : $A_{t}=A_{0} e^{\theta Z_{t}}$ où $Z_{t}$ est un vecteur de variables mesurant le niveau de développement financier et la qualité des institutions. Cependant, la définition des variables de contrôle n'est pas unique. Elle doit tenir compte des spécificités de chaque pays (Mankiw et al., 1992). Pour cette étude relative à la Côte d'Ivoire, on retient la superficie des terres agricoles (tera), l'ouverture commerciale (ouvc) et l'instabilité socio-politique (instab). Ce choix se justifie par le fait que la Côte d'Ivoire est un pays dont l'économie repose principalement sur l'agriculture d'exportation notamment le café et le cacao. En outre, l'ouverture commerciale facilite le transfert de technologies et l'étude de N'Zué (2004) montre qu'elle a un effet sur la croissance économique ivoirienne. Quant à l'instabilité socio-politique, elle constitue un frein à la croissance économique (Quenum, 2011).

La prise en compte de ces considérations permet de poser:

$$
A=\gamma e^{\beta Z}
$$

où $Z$ est le vecteur colonne des variables de contrôle et $\beta$ le vecteur ligne de leurs coefficients respectifs. 
En combinant les équations (3) et (4) et en prenant le logarithme, on obtient :

$$
\ln y=\ln \gamma+\beta Z+\alpha \ln k+(1-\alpha) \ln h
$$

La forme stochastique de cette équation donne :

$\ln y=\lambda+\beta Z+\alpha \ln k+(1-\alpha) \ln h+\varepsilon$ (6) où $\varepsilon$ est le terme de l'erreur.

En développant le produit $\beta Z$, et en posant $\ln h=\tilde{h} ; \ln k=\tilde{k} ; \ln y=\tilde{y}$ on obtient en définitive le modèle de base définissant la relation entre le produit par travailleur et les variables explicatives comme suit :

$$
\widetilde{y_{t}}=\varphi_{0}+\varphi_{1} \text { instab }_{t}+\varphi_{2} \text { Ouvc }_{t}+\varphi_{3} \text { tera }_{t}+\varphi_{4} \widetilde{k_{t}}+\varphi_{5} \tilde{h_{t}}+\varepsilon_{t}
$$

\section{II.2. Présentation des variables et sources des données}

Les données utilisées dans cette étude sont des séries annuelles allant de 1971 à 2016. Comme proxy du capital humain, on a recours à une variable stock calculée par la formule de Van Leeuwen (2007) qui elle-même est une adaptation de la méthode de Barro et Lee. Elle s'écrit:

$$
h_{t}=\left(1-\delta_{t}\right) \times h_{t-1}+\frac{L 20_{t}}{L_{t}} \times E s_{t}
$$

$\mathrm{h}_{\mathrm{t}}$ : est le stock moyen du capital humain de formation supérieure

$\delta_{t}$ est le taux de mortalité de la population à l'année $\mathrm{t}$

$\mathrm{L}_{\mathrm{t}}$ est ici la population active qui traditionnellement est âgée de 15 à 64 ans.

L20 est l'effectif de la population âgée de 20 à 24 ans, tranche d'âge normal d'inscription à l'enseignement supérieur.

Est est le taux d'enrôlement dans l'enseignement supérieur à l'année t.

Il est reproché à cette méthode de surévaluer le stock de capital humain. Pour atténuer cet effet, le second terme de la relation qui correspond au flux des nouveaux entrants a été divisé par 4 . On retient en définitive, la formule qui suit: $\quad h_{t}=\left(1-\delta_{t-1}\right) \times h_{t-1}+\frac{1}{4}\left(\frac{L 20_{t}}{L_{t}} \times E s_{t}\right)$

Les autres variables ont été calculées à partir des données extraites de la base de données de la banque mondiale sauf 1'instabilité socio-politique qui est une variable dichotomique. Elle prend la valeur 1 sur la période 2000 à 2007 et la valeur 0 ailleurs. En effet, la période 1999-2007 est marquée par des bouleversements institutionnels sans précédents dans le pays (coups d'état, suspension de la constitution, dissolution de l'assemblée nationale, rébellion armée, partition de fait du pays). Le processus de sortie de cette période continue de crise a commencé en 2007 avec le début de la réunification du pays. 
Tableau 1: Description des variables et sources des données

\begin{tabular}{|c|c|c|}
\hline Variable & Description & Sources des données \\
\hline $\mathrm{y}$ & $\begin{array}{l}\text { Niveau de produit par travailleur: } \\
\qquad y=\frac{P I B}{E F F E C T I F D E S T R A V A I L L E U R S}\end{array}$ & $\begin{array}{l}\text { Calculs de l'auteur à partir } \\
\text { des données de la banque } \\
\text { mondiale }\end{array}$ \\
\hline $\mathrm{k}$ & $\begin{array}{l}\text { Niveau de capital fixe par travailleur: } \\
\qquad k=\frac{F B C F}{\text { nombre de travailleurs }}\end{array}$ & $\begin{array}{l}\text { Calculs de l'auteur à partir } \\
\text { des données de la banque } \\
\text { mondiale }\end{array}$ \\
\hline $\mathrm{h}$ & $\begin{array}{l}\text { Stock de capital humain traduit par la proportion des } \\
\text { travailleurs de niveau d'éducation supérieur dans la } \\
\text { population active: } \\
\qquad h_{t}=\left(1-\delta_{t-1}\right) \times h_{t-1}+\frac{1}{4}\left(\frac{L 20_{t}}{L_{t}} \times E s_{t}\right)\end{array}$ & $\begin{array}{l}\text { Calculs de l'auteur, } \mathrm{h}_{0} \text { étant } \\
\text { la valeur estimée par Barro } \\
\text { et Lee pour } 1970\end{array}$ \\
\hline Ouvc & $\begin{array}{l}\text { Ouverture commerciale: } \\
\text { Ouvc }=\frac{(\text { importations }+ \text { exportations }): 2}{P I B} \times 100\end{array}$ & $\begin{array}{l}\text { Calculs de l'auteur à partir } \\
\text { des données de la banque } \\
\text { mondiale }\end{array}$ \\
\hline instab & instabilité socio-politique & Evaluation de l'auteur \\
\hline tera & $\begin{array}{l}\text { Superficie des terres agricoles par travailleur: } \\
\text { tera } \\
=\frac{\text { superficie des terres agricoles (en hectares) }}{\text { effectif des travailleurs }}\end{array}$ & $\begin{array}{l}\text { Calculs de l'auteur à partir } \\
\text { des données de la banque } \\
\text { mondiale }\end{array}$ \\
\hline
\end{tabular}

On note que l'effectif des travailleurs est obtenu en faisant le produit de la population active par le taux de participation à l'activité.

\section{II.3. Méthode d'estimation}

L'objectif principal de cette étude est de savoir si la croissance économique ivoirienne est causée en partie par l'enseignement supérieur et le cas échéant d'évaluer le niveau d'impact de celui-ci sur la croissance.

Pour ce faire, le test de causalité de Toda et Yamamoto (1995) est appliqué parce qu'il a l'avantage de prendre en compte en une seule fois plus de deux variables peu importe qu'elles soient intégrées de même ordre ou pas. La mise en oeuvre de ce test sur deux variables $y_{t}$ et $x_{t}$, se fait en considérant d'abord les fonctions $y_{t}=f\left(y_{t-i}, x_{t-i}\right)$ et $x_{t}=f\left(x_{t-i}, y_{t-i}\right)$ avec $i=$ $1,2, \ldots, m+p$ où $m+p$ est le nombre total de retards dans les deux variables. On explicite ensuite ces fonctions en les supposant linéaires et on pose les équations stochastiques ci-après :

$$
\begin{gathered}
y_{t}=\pi+\sum_{i=1}^{p+m} \alpha_{i} y_{t-i}+\sum_{i=1}^{p+m} \beta_{i} x_{t-i}+\varepsilon_{t} \\
x_{t}=\omega+\sum_{i=1}^{p+m} \gamma_{i} x_{t-i}+\sum_{i=1}^{p+m} \delta_{i} y_{t-i}+\mu_{t}
\end{gathered}
$$

$m$ est l'ordre maximum d'intégration des variables et $p$ le retard optimal. Enfin, on teste 1'hypothèse nulle $\mathrm{H}_{0}: \sum_{i=1}^{p} \beta_{i}=0$ contre 1'hypothèse alternative $\mathrm{H}_{\mathrm{a}}: \sum_{i=1}^{p} \beta_{i} \neq 0$ pour l'équation (c). On soutient que la variable $x_{t}$ cause la variable $y_{t}$ au sens de granger si l'hypothèse nulle du test est 
rejetée au seuil de signification choisi. On fait de même pour l'équation (d) en prenant comme hypothèse nulle $\mathrm{H}_{0}: \sum_{i=1}^{p} \delta_{i}=0$. En cas de rejet de $\mathrm{H}_{0}$, on conclut que $y_{t}$ cause $x_{t}$ au seuil de signification choisi.

Le test de causalité ainsi présenté, sera précédé du test de cointégration entre les variables. A cet effet, la méthode de Pesaran et al. (2001) est mise en oeuvre car elle est applicable sur les séries multivariées sans exiger qu'elles soient intégrées de même ordre. Ce test s'appuie sur une spécification en modèle ARDL (modèle auto régressif à retards distribués) cointégré. La régression ARDL est utilisée pour identifier les relations de long terme et celles de court terme. Dans cette étude, le modèle ARDL applicable à partir de l'équation (7) qui définit le PIB par travailleur comme variable endogène est le suivant :

$\Delta \widetilde{y_{t}}=\beta_{0}+\sum_{i=1}^{m} \beta_{1 i} \Delta \tilde{y}_{t-i}+\sum_{i=0}^{n} \beta_{2 i} \Delta$ instab $_{t-i}+\sum_{i=0}^{p} \beta_{3 i} \Delta O u v c_{t-i}+$ $\sum_{i=0}^{q} \beta_{4 i} \Delta \operatorname{tera}_{t-i}+\sum_{i=0}^{r} \beta_{5 i} \Delta \tilde{k}_{t-i}+\sum_{i=0}^{s} \beta_{6 i} \Delta \tilde{h}_{t-i}+\alpha_{1} \tilde{y}_{t-1}+$ $\alpha_{2}$ instab $_{t-1}+\alpha_{3}$ Ouvc $_{t-1}+\alpha_{4}$ tera $_{t-1}+\alpha_{5} \tilde{k}_{t-1}+\alpha_{6} \tilde{h}_{t-1}+\varepsilon_{t}$

On note que $\Delta$ indique l'opérateur de différence première, $\varepsilon_{t}$ est le terme de l'erreur qui est ici un bruit blanc; $m, n, p, q, r$ et $s$ représentent dans chaque terme correspondant le nombre maximum de retards retenu par le critère d'information choisi qui, le plus souvent, est le critère d'Akaike (AIC) ou celui de Schwarz (SIC). $\beta_{0}$ est la constante, les paramètres $\alpha_{i}$ caractérisent l'équilibre de long terme et enfin les paramètres $\beta_{i}$ définissent les relations de court terme entre les variables. Le test de cointégration aux bornes de Pesaran et al. (2001) considère comme hypothèse nulle $H_{0}: \alpha_{1}=\alpha_{2}=\cdots=\alpha_{n}=0$ qui traduit une absence de cointégration; contre l'hypothèse alternative $H_{1}: \alpha_{1} \neq \alpha_{2} \neq \cdots \neq \alpha_{n} \neq 0$ qui marque l'existence d'une relation de long terme (une cointégration) entre les variables.

\section{Presentation des Resultats \\ III.1. Evolution graphique des variables}

Les figures $1 \& 2$ donnent un aperçu graphique de l'évolution des variables de l'étude sur la période 1971-2016. 


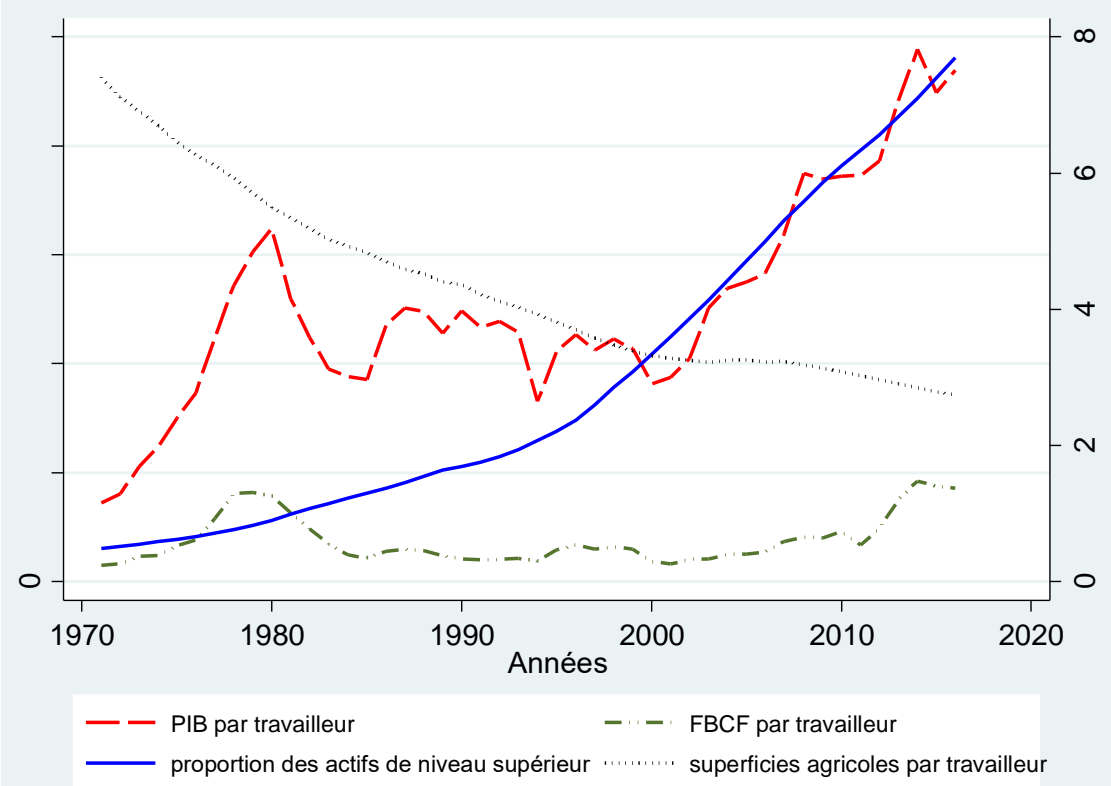

Figure 1: Evolution des variables PIB, FBCF, superficies agricoles par travailleur et de la proportion des actifs de niveau supérieur de 1971 à 2016

Le produit intérieur brut (PIB) et la formation brute du capital fixe (FBCF) par travailleur sont exprimés en dollars courants US et se lisent sur l'axe des ordonnées à gauche. On constate que la décennie 1970-1980 considérée comme la décennie du miracle économique ivoirien est marquée par la hausse exponentielle à la fois du PIB et des investissements en capitaux fixes. Ces investissements chutent au début des années 1980 qui coïncide avec le début de la crise économique dans le pays et la mise en œuvre des programmes d'ajustement structurel. La formation brute du capital fixe ne décolle véritablement à nouveau qu'à partir de 2012 à un rythme semblable à celui de la décennie 1970-1980. Toutefois, la croissance du Pib par travailleur a repris depuis le début des années 2000.

La proportion des actifs de niveau supérieur et la superficie des terres agricoles exprimée en hectares se lisent sur l'axe des ordonnées à droite. Il apparait que la proportion de la population active ayant atteint au moins le niveau de l'enseignement supérieur augmente de façon continue avec une pente plus accrue après 1994, soit deux ans après la création des centres universitaires de Bouaké et d'Abobo-Adjamé transformés en universités autonomes en 1995. On note une baisse continue de la superficie des terres agricoles par travailleur sur la période 1971-2016 quand bien même l'économie du pays repose sur l'agriculture. De près de 8 hectares par travailleur en 1971 on est passé à moins de 3 hectares de terres agricoles par travailleur en 2016. On constate cependant un plateau dans cette baisse entre 
2002 et 2007, période où le pays était divisé en deux du fait de la rébellion armée.

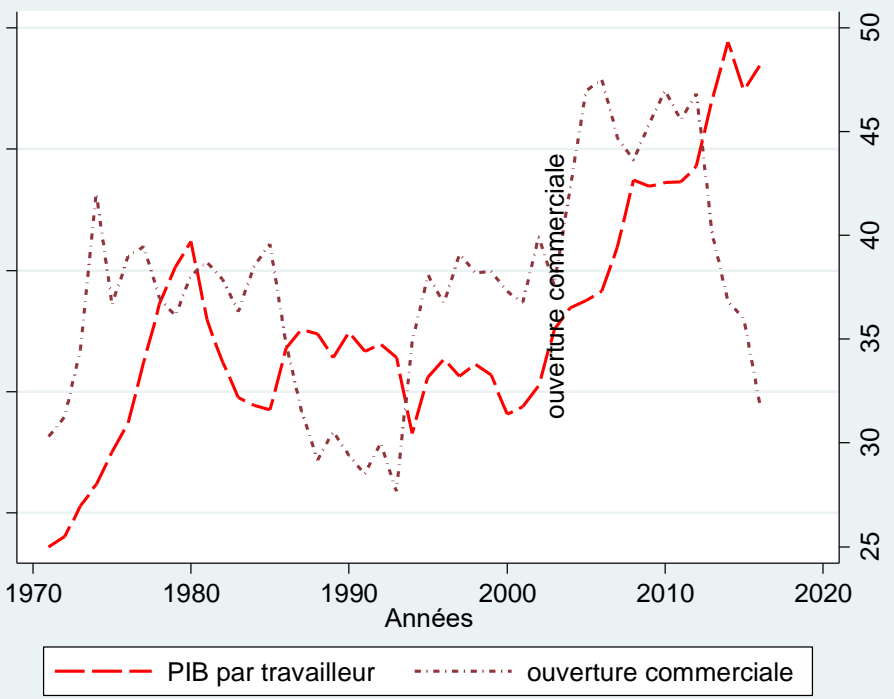

Figure 2: Evolution du PIB par travailleur et du niveau d'ouverture commerciale du pays de 1971 à 2016

Le taux d'ouverture du pays est très instable sur la période de l'étude. Il vacille entre $35 \%$ et $42 \%$ jusqu'à 1986 . Il connait une baisse considérable à partir de 1987 où les exportations agricoles furent durement éprouvées par la sécheresse qui a touché la moitié du pays. Il varie ensuite autour de 30\% jusqu'à 1993. A partir de 1994, date de la première dévaluation du franc CFA, le taux d'ouverture retrouve son niveau d'avant la chute de 1987. Il garde ce niveau jusqu'en 2005 où il franchit un nouveau palier pour se situer autour de 45\%. Depuis 2012, on assiste à une baisse continue et remarquable du taux d'ouverture du pays. En général, une baisse du taux d'ouverture n'est pas nécessairement un repli des échanges avec l'extérieur. Même si elle peut être l'effet d'une chute des exportations, elle traduit dans bien des cas une augmentation relative du niveau du PIB à l'instar de ce qui est observé depuis 2012.

\section{III.2. Description sommaire des variables}

Le tableau ci-après donne les principales caractéristiques des variables telles qu'elles ont été utilisées dans les estimations. 
Tableau 2 : Statistiques descriptives des variables

\begin{tabular}{cccccccc}
\hline Variable & Obs & Min & Max & Mean & $\begin{array}{c}\text { Std. } \\
\text { Dev. }\end{array}$ & Skewness & Kurtosis \\
\hline$\tilde{y}$ & 46 & 6,579 & 8,494 & 7,769 & 0,412 & $-0,701$ & 4,022 \\
\hline$\tilde{k}$ & 46 & 5,017 & 6,827 & 5,803 & 0,510 & 0,616 & 2,333 \\
\hline$\tilde{h}$ & 46 & $-0,717$ & 2,040 & 0,747 & 0,854 & $-0,085$ & 1,762 \\
\hline ouvc & 46 & 27,674 & 47,535 & 37,584 & 5,299 & 0,072 & 2,422 \\
\hline tera & 46 & 2,738 & 7,405 & 4,325 & 1,327 & 0,766 & 2,434 \\
\hline instab & 46 & 0 & 1 & 0,174 & 0,383 & 1,721 & 3,961
\end{tabular}

Note: $\tilde{y}=\ln$ (pib/travailleur); $\tilde{k}=\ln ($ formation brute du capital fixe/travaileur);

$\tilde{h}=\ln$ (proportion de la population active de niveau supérieur)

On constate une forte dispersion du capital humain $(\tilde{h})$, de la superficie des terres agricoles par travailleur (tera) à cause de leurs écarts types relativement élevés au regard des moyennes correspondantes. Néanmoins, tous les coefficients d'asymétrie (skewness) sont compris entre -1 et +1 (mise à part l'instabilité), ce qui est acceptable pour une distribution normale selon Hair Jr. et al. (2014). Par contre les coefficients d'aplatissement indiquent l'existence de valeurs élevées pour le pib (kurtosis largement supérieur à 3 ) et des valeurs beaucoup plus faibles par rapport à la distribution normale pour le capital humain (kurtosis très inférieur à 3$)^{1}$.

\section{III.3. Tests de stationnarité}

La stationnarité est un préalable à la modélisation des séries temporelles afin d'éviter des régressions illusoires. Pour vérifier si les variables de l'étude sont stationnaires, le test ADF (Dickey Fuller augmenté) et sa version généralisée DF-GLS ont été utilisés et complétés par le test KPSS (Kwiatkowski-Phillips-Schmidt-Shin) qui a une procédure différente. Ce test adopte comme hypothèse nulle la non stationnarité des variables contrairement aux deux précédents. Les résultats sont donnés dans le tableau qui suit.

${ }^{1}$ Sous le logiciel Stata, le kurtosis d'une variable aléatoire normalement distribuée est 3 et son skewness est 0 . 
Tableau 3: Résultats des tests de stationnarité

\begin{tabular}{|c|l|l|l|l|l|l|}
\hline \multirow{2}{*}{ variable } & \multicolumn{3}{|c|}{ En niveau : I(0) } & \multicolumn{2}{c|}{ En différence première : I(1) } \\
\cline { 2 - 7 } & ADF & DF-GLS & KPSS & ADF & DF-GLS & KPSS \\
\hline$\tilde{y}$ & $-2,626$ & $\mathbf{- 3 , 6 4 9} * *$ & $\mathbf{0 , 3 6 2} * * *$ & $\mathbf{- 4 , 7 4 2} * *$ & $\mathbf{- 3 , 8 3 0} * *$ & $\mathbf{0 , 2 1 7} * * *$ \\
\hline$\tilde{k}$ & $-1,276$ & $-2,804$ & $\mathbf{0 , 5 1 9} * * *$ & $\mathbf{- 4 , 4 9 0} * * *$ & $\mathbf{- 3 , 6 9 7} * *$ & $\mathbf{0 , 1 8 5} * *$ \\
\hline$\tilde{h}$ & $\mathbf{- 2 , 9 7 0} * *$ & $-2,695$ & $\mathbf{0 , 4 0 2} * * *$ & $-1,505$ & $-2,733$ & $\mathbf{0 , 3 2 7} * * *$ \\
\hline Ouvc & $-2,053$ & $-2,574$ & 0,036 & $\mathbf{- 6 , 2 6 0} * * *$ & $\mathbf{- 3 , 7 3 9} * *$ & 0,108 \\
\hline tera & $\mathbf{- 1 2 , 8 3 2} * * *$ & $-0,739$ & $\mathbf{1 , 0 6} * * *$ & $-2,857$ & $-1,191$ & $\mathbf{0 , 3 5 4} * * *$ \\
\hline instab & $-1,881$ & -1.796 & $\mathbf{0 , 3 2 7} * * *$ & $\mathbf{- 6 , 4 8 1} * * *$ & $\mathbf{- 4 , 0 8 7} * * *$ & 0,0706 \\
\hline & & & & & & \\
\hline Valeurs critiques & $-2,944$ & $-3,190$ & 0,146 & $-2,947$ & $-3,190$ & 0,146 \\
\hline
\end{tabular}

**réponse favorable au seuil de $5 \%$; *** réponse favorable au seuil de $1 \%$

L'analyse des critères montre que les variables $\tilde{y}, \tilde{k}$, ouvc et instab sont intégrées d'ordre $1[\mathrm{I}(1)]$ tandis que les variables $\tilde{h}$ et tera sont intégrées à niveau $[\mathrm{I}(0)]$. Les variables intégrées à niveau sont stationnaires c'est-à-dire qu'elles ont une moyenne et une variance constantes dans le temps. Ce qui n'est pas le cas pour les variables intégrées d'ordre 1 qui doivent être différenciées une fois pour être stables en moyenne et en variance. Une régression entre ces variables est donc soumise à la validation préalable d'un test de cointégration pour vérifier l'éventualité d'une relation d'équilibre de longue durée entre elles. Avant cela, on détermine le décalage optimal des valeurs des variables. C'est le décalage qui permet d'obtenir après estimation des résultats statistiquement significatifs avec le moins de paramètres.

\section{III.4. Choix du nombre optimal de retards}

La procédure de sélection du nombre de retards mise en œuvre sous STATA a donné les résultats ci-après.

Tableau 4: Choix de l'ordre des retards

\begin{tabular}{|c|c|c|c|c|c|c|}
\hline \multicolumn{7}{|c|}{$\begin{array}{c}\text { Variables dépendantes }: \widetilde{y} \widetilde{\boldsymbol{k}} \widetilde{h} \text { ouvc tera instab } \\
\text { Variable exogène }: \text { constante }\end{array}$} \\
\hline Lag & $\mathbf{L L}$ & LR & FPE & AIC & HQIC & SBIC \\
\hline 0 & $-163,629$ & - & 0,00013 & 8,07755 & 8,16854 & 8,32579 \\
\hline 1 & 236,6 & 800,46 & $3,9 \mathrm{e}-12$ & $-9,26669$ & $-8,62976^{*}$ & $-7,52902$ \\
\hline 2 & 258,569 & 43,937 & $8,4 \mathrm{e}-12$ & $-8,59851$ & $-7,41565$ & $-5,37141$ \\
\hline 3 & 309,355 & 101,57 & $5,6 e-12$ & $-9,30261$ & $-7,57381$ & $-4,58608$ \\
\hline 4 & 374,126 & $129,54 *$ & $2,8 \mathrm{e}-12 *$ & $-10,6727 *$ & $-8,39796$ & $-4,46672$ \\
\hline
\end{tabular}

*le nombre de retards choisi par le critère

Trois (3) des cinq (5) critères en présence dans cette analyse ont sélectionné 4 comme le nombre optimal de retard. Ainsi, l'effet d'une variable exogène pourra être capté jusqu'à quatre années plus tard sur la variable dépendante. 


\section{III.5. Test de cointégration}

Les variables étant intégrées à des ordres différents, le test de cointégragtion qui convient est celui de Pesaran et al. (2001). Ce test a été mis en oeuvre en utilisant les valeurs critiques développées par Kripfganz et Schneider (2019) pour les échantillons de petite taille. Les résultats obtenus sont les suivants:

Tableau 5: Résultats du test de cointégration aux bornes

\begin{tabular}{|c|c|c|c|c|c|c|}
\hline \multicolumn{7}{|c|}{$\tilde{y}$ Variable dépendante : } \\
\hline \multicolumn{3}{|c|}{ Statistiques calculées : } & 7,707 & $-10,119$ & \multirow{2}{*}{\multicolumn{2}{|c|}{$\begin{array}{l}\text { cas } 3 \\
\text { p-value }\end{array}$}} \\
\hline \multirow{2}{*}{ Statistique } & \multicolumn{2}{|c|}{ Valeurs critiques à 5\% } & \multicolumn{2}{|c|}{ Valeurs critiques à $1 \%$} & & \\
\hline & $\mathrm{I}(0)$ & $\mathrm{I}(1)$ & $\mathrm{I}(0)$ & $\mathrm{I}(1)$ & $\mathrm{I}(0)$ & $\mathrm{I}(1)$ \\
\hline $\mathrm{F}$ & 2,927 & 4,542 & 4,175 & 6,272 & 0,000 & 0,000 \\
\hline $\mathrm{t}$ & $-2,823$ & $-4,203$ & $-3,586$ & $-5,117$ & 0,000 & 0,000 \\
\hline
\end{tabular}

L'hypothèse nulle de ce test est l'absence de cointégration. La p-value est inférieure au seuil critique même à $1 \%$ aussi bien pour les variables $\mathrm{I}(0)$ que pour les variables I(1). On rejette donc l'hypothèse nulle d'absence de cointégration entre les variables. Autrement dit, on admet qu'il existe au moins une relation de long terme qui exprime la variable endogène, le produit par travailleur, en fonction des variables exogènes.

\section{III.6. Relations de long terme}

Les estimations du modèle ARDL a donné les résultats suivants.

Tableau 6: Coefficients de long terme

\begin{tabular}{|c|c|c|c|}
\hline \multicolumn{4}{|c|}{$\begin{array}{c}A R D L(4,0,4,1,0,2) \text { choisi selon le critère bic } \\
\text { Variable dépendante }: \text { produit intérieur brut par travailleur }(\tilde{y}) \\
R^{2}=0,9143 \quad F(16,25)=101,34 \\
\text { Adj- } R^{2}=0,8595 \\
\text { Prob }>F=0,0000\end{array}$} \\
\hline Variable indépendante & Coefficient & t-statistic & p-value \\
\hline $\operatorname{Adj} \tilde{y}(-1)$ & $-0,633$ & $-10,12$ & 0,000 \\
\hline$\tilde{k}$ & 0,596 & 11,13 & 0,000 \\
\hline$\tilde{h}$ & $-1,063$ & $-4,50$ & 0,000 \\
\hline Ouvc & 0,016 & 2,84 & 0,009 \\
\hline tera & $-0,819$ & $-5,43$ & 0,000 \\
\hline instab & 0,261 & 3,79 & 0,001 \\
\hline Constante & 5,407 & 9,82 & 0,000 \\
\hline
\end{tabular}

On constate que le coefficient d'ajustement ou la force de rappel ($0,633$ ) est négatif et significatif même à $1 \%$ (p-value $=0,000)$. Cela confirme l'existence d'une relation de long terme entre la variable expliquée et les variables exogènes. On en déduit que les variations du PIB par travailleur au cours d'une année sont corrigées à 63,30\% l'année qui suit. La p-value associée à la statistique de Fisher montre que le modèle est globalement significatif à $95 \%$. De plus, toutes les variables ont un impact significatif à 
long terme sur le niveau global du produit. Suivant le $\mathrm{R}^{2}$ ajusté, elles expliquent à près de $86 \%$ les variations du PIB par travailleur. On note aussi qu'une augmentation de $1 \%$ du stock de capital fixe par travailleur entraîne un accroissement de $0,596 \%$ du produit global. Cependant, un accroissement de $1 \%$ de stock de capital humain entraîne une baisse de $1,06 \%$ du produit global.

\section{III.7. Dynamique de court terme}

Les estimations montrent que le niveau de produit par travailleur, à court terme, subit négativement à différents degrés, les augmentations du stock de capital humain, du niveau d'ouverture de l'économie et l'instabilité sociopolitique. Cependant, le stock de capital physique ne semble pas avoir significativement d'effets immédiats sur le niveau du produit.

Tableau 7: Coefficients significatifs à court terme

\begin{tabular}{|c|c|c|c|}
\hline Variable & Coefficient & t-statistique & p-value \\
\hline$\Delta \widetilde{h}$ & $-6,618$ & $-5,02$ & 0,000 \\
\hline$\Delta o u v c$ & $-0,017$ & $-4,46$ & 0,000 \\
\hline Ainstab & $-0,214$ & $-4,88$ & 0,000 \\
\hline
\end{tabular}

\section{III.8. Tests de diagnostic}

Pour tester la validité des estimations, différents tests ont été mis en oeuvre. Les résultats de ces tests sont présentés dans le tableau 8.

Tableau 8: Diagnostic des résidus

\begin{tabular}{|c|c|c|}
\hline Test & Chi $^{2}$ & Prob > Chi \\
\hline Normality test & 56,96 & 0,5140 \\
\hline Heteroskedasticity & 42,00 & 0,4274 \\
\hline Breusch Godfrey LM test & 0,084 & 0,7714 \\
\hline Breusch-Pagan & 0,80 & 0,3720 \\
\hline Durbin's alternative & 0,048 & 0,8260 \\
\hline & Statique F & Prob > F \\
\hline Ramsey RESET & 1,23 & 0,3239 \\
\hline
\end{tabular}

Il ressort de ces tests que les résidus sont normalement distribués et il n'y a ni autocorrélation ni hétéroscédasticité. De plus, le test de Ramsey RESET montre que le modèle ne souffre pas d'une mauvaise spécification. En somme, on peut affirmer que le modèle est valide et les estimations robustes. Cependant, les tests de cusum et cusumq révèlent une sensibilité du modèle aux périodes de crise socio-politique.

\section{III.9. Test de stabilité du modèle}

Les tests de cusum et cusumq permettent de vérifier la stabilité à long terme d'un modèle après régression ARDL. Le cusum analyse la stabilité de la constante tandis que le cusumq vérifie en particulier la stabilité des coefficients. 


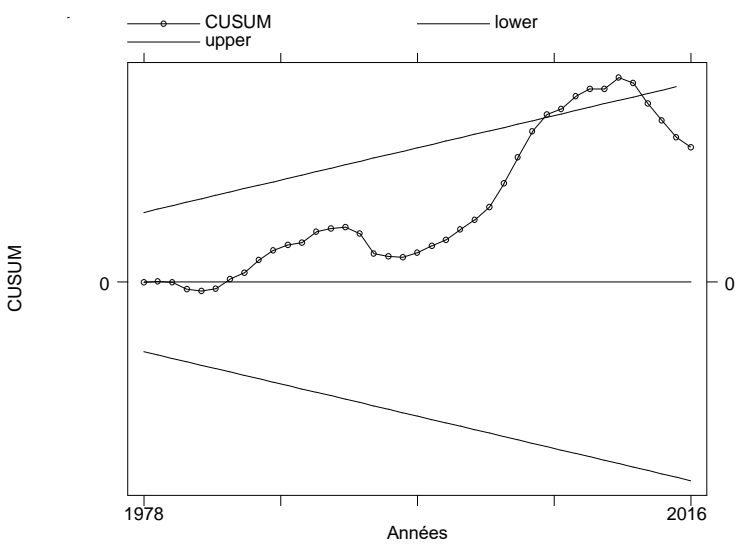

Figure 1: Résultat du test de cusum CUSUM squared

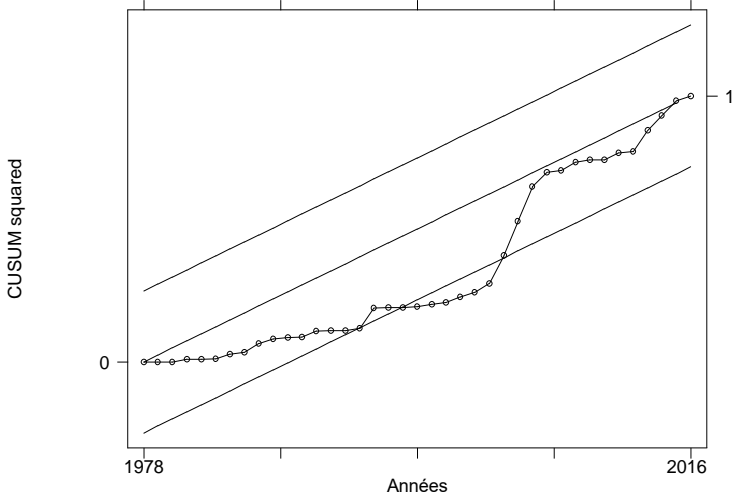

Figure 2: Résultat du test de cusumq

Ces deux tests indiquent des périodes d'instabilité pour le modèle. Les coefficients du modèle sont affectés par la période de crise sociopolitique qui a commencé avec le coup d'état de 1999 et s'est aggravée avec la rébellion de 2002. On note que les coefficients retrouvent leur statbilité à partir de 2007 date à laquelle il y a eu la flamme de la paix et le début de réunification du pays. Après cette période, on entre dans une phase de sortie de crise qui se prolonge jusqu'en 2012. Cette phase est marquée par une instabilité de la constante (voir résultat cusum).

\section{III.10. Test de causalité de Toda-Yamamoto}

On a vu que les variables sont intégrées à l'ordre $1(\mathrm{~m}=1)$ et le retard optimal est $4(\mathrm{p}=4)$. Le nombre maximum de retards à considérer dans l'équation (c ) ou (d) du test de Toda et Yamamoto (1995) est $m+p=5$. Les résultats sont présentés dans le tableau qui suit. 
Tableau 9: Résultats du test de causalité de Toda-Yamamoto

\begin{tabular}{|c|c|c|c|}
\hline Hypothèse nulle & $\begin{array}{l}\text { Statistique } \\
\text { du Chi }^{2}\end{array}$ & Prob> chi ${ }^{2}$ & $\begin{array}{c}\text { Causalité au sens de } \\
\text { Granger }\end{array}$ \\
\hline$\tilde{y}$ ne cause pas $\tilde{k}$ & 12,23 & $0,0157 * *$ & \multirow{2}{*}{$\begin{array}{l}\text { Causalité } \\
\text { unidirectionnelle } \\
\tilde{y}\end{array}$} \\
\hline$\tilde{k}$ ne cause pas $\tilde{y}$ & 7,86 & 0,0967 & \\
\hline$\tilde{y}$ ne cause pas $\tilde{h}$ & 74,93 & $0,0000 * * *$ & \multirow{2}{*}{$\begin{array}{l}\text { Causalité bidirectionnelle } \\
\longrightarrow \\
\widetilde{y}\end{array}$} \\
\hline$\widetilde{h}$ ne cause pas $\tilde{y}$ & 16.14 & $0,0028 * * *$ & \\
\hline$\tilde{h}$ ne cause pas $\tilde{k}$ & 38,73 & $0,0000 * * *$ & \multirow{2}{*}{ 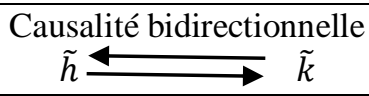 } \\
\hline$\tilde{k}$ ne cause pas $\tilde{h}$ & 60,15 & $0,0000 * * *$ & \\
\hline
\end{tabular}

Il apparait une relation de causalité réciproque entre le stock de capital humain et le niveau de produit par travailleur. Le coefficient du stock de capital humain étant négatif, l'enseignement supérieur peut être considéré comme un frein à la croissance du produit global en Côte d'Ivoire. Cependant, il ressort de ce test que le niveau de produit par travailleur cause à sens unique le stock de capital fixe. Il semble ainsi que c'est plutôt la croissance économique qui conduit à l'achat des équipements et à l'investissement en capitaux fixes. Par ailleurs, le test indique une causalité réciproque entre les investissements en capitaux fixes et la formation du stock de capital humain de niveau supérieur. Autrement dit, l'emploi des travailleurs de niveau supérieur conduit à une utilisation accrue des nouveaux équipements et outils de travail. De même, l'achat d'équipements et outils modernes et performants provoque un accroissement de la demande des travailleurs de formation supérieure.

\section{III.11. Analyse de l'intensité causale entre l'enseignement supérieur et la croissance économique}

Le test de causalité a montré une réciprocité dans la relation de causalité entre l'enseignement supérieur et la croissance économique. Selon Taleb et Bates (2004, p.14), il est important d'analyser l'intensité de chaque direction causale lorsqu'il apparait une causalité bidirectionnelle entre deux (02) variables d'intérêt afin de mettre en évidence les interprétations théoriques qui sont le plus empiriquement valides. Ils définissent donc un indicateur d'intensité causale (IC) qu'on peut adjoindre au test de causalité de Toda et Yamamoto (1995). Il se calcule avec la formule suivante :

$$
\text { IC }=(1-\text { pvalue }) *|\varepsilon|
$$

Où pvalue est obtenue lors du test de causalité au seuil $\alpha$ de significativité fixé. $(1-p v a l u e)$ est considéré comme le degré de présomption de causalité entre les variables tandis que $\varepsilon$ est l'élasticité de la variable causale vers la variable causée. Lorsque les variables sont prises en logarithme, l'élasticité d'une variable $\mathrm{Y}$ par rapport à la variable $\mathrm{X}$ se calcule par la formule: 


$$
\varepsilon_{X \rightarrow Y}=\frac{\partial \ln Y}{\partial \ln X}
$$

Dans le modèle ARDL estimé, le niveau de produit par tête et le stock de capital humain sont pris en logarithme. Ce qui signifie que les coefficients de ces variables représentent des élasticités. Ainsi, les élasticités utilisées pour le calcul de $I C$ sont les coefficients de long terme obtenus après estimation du modèle ardl. A la suite des calculs, il a été obtenu le tableau qui suit :

Tableau 10: Intensités causales entre enseignement supérieur et croissance

\begin{tabular}{|c|c|c|c|}
\hline Sens de la causalité & pvalue & Elasticité & Indice de causalité (IC) en \% \\
\hline$\tilde{h}$ & 0,0028 & $-1,063$ & 106,00 \\
\hline$\tilde{y} \longrightarrow \tilde{h}$ & $\mathbf{0 , 0 0 0 0}$ & $-0,9407$ & 94,07 \\
\hline
\end{tabular}

Il apparait donc que l'enseignement supérieur a plus d'impact sur la croissance que celle-ci n'en a sur l'enseignement supérieur. Seulement, cet impact est négatif.

\section{Discussion}

L'un des résultats majeurs de ce travail est que l'enseignement supérieur traduit ici par la proportion de la population active ayant atteint le niveau supérieur a un impact négatif et significatif sur la croissance du PIB en Côte d'Ivoire, à court terme et à long terme. Ce résultat présente des divergences avec plusieurs travaux qui ont porté sur l'éducation et la croissance économique de ce pays. Il diverge du résultat de Keho (2009) qui a trouvé que le capital humain en Côte d'Ivoire, approché par les dépenses publiques d'éducation, n'a pas eu de contribution significative à l'accroissement de la richesse nationale sur la période 1980 - 2005. Dans son étude, l'auteur a eu recours à la méthode du maximum de vraisemblance et au test de cointégration de Johansen (1988) pour analyser l'effet de long terme des dépenses publiques d'éducation et de santé sur le PIB par tête du pays. Le résultat s'oppose à celui de Coulibaly (2013). En effet, cet auteur a étudié la relation entre les dépenses publiques d'éducation et la croissance économique en Côte d'Ivoire sur la période 1970 - 2005, en utilisant le taux de croissance du PIB comme variable endogène. En appliquant un modèle à correction d'erreur, il trouve qu'à court terme, un accroissement marginal des dépenses publiques d'éducation conduit à une augmentation d'environ 5,8\% du taux de croissance du PIB. Le présent résultat est plutôt conforme à celui de Kouton (2018) en considérant seulement les effets de long terme. L'étude de Kouton (op. cit.) porte sur une période relativement plus longue que celles des précédents auteurs. Il utilise une série de données annuelles allant de 1970 à 2015, en mesurant la croissance économique, variable dépendante, par le PIB réel. Il évalue l'impact des dépenses publiques d'éducation, exprimées en 
pourcentage du PIB, sur la variable dépendante en adoptant un modèle autorégressif à retards échelonnés (modèle ARDL) et le test de causalité de Toda et Yamamoto (1995). Les résultats qu'il trouve indiquent qu'à long terme, les dépenses publiques d'éducation ont un effet négatif et significatif sur la croissance économique. Certes, un effet positif apparaît à court terme mais, contrairement à l'étude de Coulibaly (2013), il n'est pas significatif. En somme, cette étude montre que les dépenses publiques d'éducation, au mieux, n'entraînent pas la croissance économique en Côte d'Ivoire. Outre ce résultat, elle présente des similitudes avec le présent article en ce qui concerne la période d'étude (1970-2016) et les méthodes d'analyse à savoir l'usage du modèle ARDL et le test de causalité de Toda et Yamamoto.

Cependant, ces précédents travaux sur l'éducation et la croissance économique en Côte d'Ivoire, présentent ensemble une faiblesse. En effet, elles évaluent l'effet des dépenses publiques d'éducation sur la croissance économique. Or, l'utilisation des dépenses publiques comme proxy du capital humain peut surestimer l'impact de celui-ci sur la croissance. Car, d'une part, la théorie keynésienne considère l'ensemble des dépenses publiques quelle qu'en soit la nature comme facteur de croissance économique grâce au mécanisme du multiplicateur. Ce constat a été fait par Urhie (2014) dans son étude portant sur le Nigéria. L'auteur relève que les dépenses courantes d'éducation ont un important effet sur la croissance dû à l'effet multiplicateur. On pourrait comprendre ainsi les effets positifs de court terme constatés dans les études de Coulibaly (2013) et Kouton (2018). D'autre part, il se pose le problème de l'efficacité des dépenses publiques. Le montant des ressources financières allouées à l'éducation ne se traduit pas forcément en niveau de scolarisation ou d'acquisitions des apprenants. D'ailleurs Urhie (op. cit.) souligne ce contraste en observant que les dépenses publiques consacrées à l'éducation au Nigeria induisent la croissance économique pendant qu'elles n'améliorent pas le niveau d'éducation. Il est donc préférable de recourir à d'autres variables que des variables financières, pour capter l'effet réel du capital humain sur la croissance économique. Par ailleurs, aucun de ces précédents travaux n'étudie l'impact spécifique de l'enseignement supérieur sur la croissance économique en Côte d'Ivoire. Si on admet que l'éducation influe la croissance économique, il apparait que tous les niveaux du système éducatif ne présentent pas toujours le même effet. Ainsi, le présent travail se distingue des précédents travaux sur l'éducation et la croissance économique en Côte d'Ivoire par trois éléments. Le premier est qu'il utilise une variable stock comme proxy du capital humain plutôt qu'une variable monétaire. Le deuxième est l'utilisation du produit par travailleur qui correspond mieux à la fonction de production à l'origine du modèle économétrique au lieu du produit par tête. Et le troisième élément est que ce travail s'intéresse particulièrement à l'impact de l'enseignement supérieur sur la croissance. 
Le fait que cet impact soit négatif était une hypothèse fort plausible. En effet, certaines conditions sont jugées nécessaires pour que l'éducation favorise la croissance économique. En se référant à Prichett (2001), on peut en retenir trois. D'abord, il faut un environnement institutionnel et une gouvernance de bonne qualité, à défaut, le stock de capital humain éducatif peut conduire à un ralentissement de la croissance économique, les élites mettant leurs compétences au service de la corruption, des détournements et des vices en général. Ensuite, il faut une utilisation effective et adéquate des travailleurs éduqués. Quand ce n'est pas le cas, on assiste à une baisse considérable des rendements marginaux de l'éducation. Enfin, la qualité de l'éducation doit être bonne. Lorsque le système éducatif est de mauvaise qualité, l'accumulation des années d'études ou des niveaux scolaires ne correspond pas à une accumulation réelle de stocks de connaissances ou de compétences. Ainsi, le nombre d'années d'études pourrait surestimer le stock réel de capital humain. Cependant, la situation de la Côte d'Ivoire et de l'enseignement supérieur ivoirien ne semble pas conforme à ces conditions. Premièrement, la qualité de l'environnement institutionnel paraît insuffisante avec un niveau assez élévé de corruption (Keho, 2012, p.24). Deuxièmement, l'enseignement supérieur est le niveau d'enseignement qui en Côte d'Ivoire, connait le plus fort taux de chômage des diplômés (Ramanantoanina, 2017). L'ampleur et la durée de ce chômage pourraient expliquer aussi l'écart entre l'effet de court terme $(-6,62 \%)$ et celui de long terme $(-1,06 \%)$ constatés dans le résultat actuel. En réalité le chômage est plus important à court terme chez les diplômés mais ils finissent après une certaine période par trouver un emploi qu'il soit en adéquation ou pas avec leur formation, permettant ainsi une revalorisation de leurs rendements. Troisièmement, l'enseignement supérieur ivoirien vit une situation de crise depuis les années 1990 qui a conduit à une forte dégradation de la qualité de ce niveau du système éducatif (Ministère de l'Enseignement Supérieur et de la Recherche Scientifique, nd). Par ailleurs, des résultats similaires ont déjà été trouvés par Benhabib et Spiegel (1994), Prichett (2001). Ces auteurs avaient relevé des liens négatifs entre l'éducation et la croissance économique. On remarque qu'ils ont tous utilisé un modèle économétrique issu de la fonction de production de type Cobb-Douglas et une variable stock du capital humain à l'instar du présent travail.

Concernant le cas spécifique de l'enseignement supérieur, les résultats de ce travail sont contraires à ceux des études portées sur des pays développés ou économiquement avancés.

Au niveau des pays développés, plusieurs études indiquent que l'enseignement supérieur a effectivement un impact positif sur la croissance économique. L'étude de Holland et al. (2013) est un exemple porté sur un échantillon de 15 pays de l'OCDE sur la période 1982 - 2005. En utilisant un 
modèle à correction d'erreur, ces auteurs trouvent qu'un accroissement marginal de la proportion des travailleurs de niveau supérieur dans la force de travail, conduit à une augmentation de 0,2 à $0,5 \%$ du produit par heure de travail. Ce résultat est corroboré par les travaux d'Armeanu et al. (2018). Ceux-ci ont examiné les facteurs de croissance du PIB réel dans les 28 pays de l'Union Européenne. Ils trouvent que l'enseignement supérieur, représenté par les dépenses par étudiant affecte positivement et durablement la croissance économique.

En outre, des études mettant ensemble plusieurs pays de niveaux de développement différents étayent également l'hypothèse que l'enseignement supérieur est facteur de croissance économique pour les pays avancés. Parmi elles, nous avons les travaux de Jihène (2015). S'intéressant à la causalité entre l'enseignement supérieur et la croissance économique en Tunisie, Maroc et Corée du Sud, l'auteur trouve que seule la Corée du Sud qui présente une situation économique avancée, fait apparaitre une cointégration entre ces deux variables.

Des travaux portant sur les pays intermédiaires ou émergents indiquent aussi l'importance de l'enseignement supérieur dans la croissance de ces pays. En Inde, par exemple, même si la croissance est portée par tous les niveaux du système éducatif, c'est l'enseignement supérieur qui l'impacte le plus (Kotaskova et al., 2018).

Par contre, les études portées particulièrement sur les pays en développement donnent des résultats assez variés.

Nowak et Dahal (2016) ont analysé le lien entre éducation et croissance économique au Népal. Dans leur recherche qui couvre la période 1975 - 2014, les auteurs représentent le capital humain par les différents taux de scolarisation (primaire, secondaire, supérieur) et la variable économique dépendante par le PIB. En utilisant la méthode des moindres carrés ordinaires (MCO), ils trouvent que les niveaux d'enseignement secondaire et primaire contribuent plus à la croissance économique au Népal que ne fait le niveau d'enseignement supérieur.

En nous intéressant à la zone Afrique, les résultats sont plutôt mitigés. Les études menées par Gymiah-Brempong, Paddison et Mitiku (2006) indiquent que globalement, tous les niveaux d'éducation ont un impact positif et significatif sur la croissance du revenu par tête du continent. Cependant, l'enseignement supérieur se démarque nettement avec un impact beaucoup plus élevé. Cette étude a porté sur un panel de 34 pays africains sur la période 1960 - 2000. Elle est confirmée par Gymiah-Brempong (2010) avec un échantillon plus large de pays (52 pays sur les 54 d'Afrique) et une période d'étude plus grande $(1960$ - 2010). Celui-ci relève l'importance de l'enseignement supérieur par rapport aux autres niveaux d'enseignement pour la croissance du revenu en Afrique. Ces études à caractère global cachent, 
cependant, une diversité de situations dans l'ensemble des pays. Déjà, l'étude de Quenum (2011) indiquait que les niveaux d'éducation post-primaires produisent significativement un effet négatif sur la croissance économique des pays de 1'Union Economique et Monétaire Ouest Africaine (UEMOA). Les cas de la Tunisie et du Maroc traités dans les travaux de Jihène (2015) révèlent que l'enseignement supérieur n'est pas déterminant dans la croissance de ces deux pays. C'est aussi le cas au Burkina si on se réfère à Kafimbou et al. (2011). Ces auteurs estiment qu'il n'y a pas d'évidences pour soutenir que l'enseignement supérieur influence la croissance économique au Burkina Faso. Par contre, Fonkeng et Ntembe (2009) ont relevé, par la méthode des moindres carrés ordinaires, un impact positif et significatif du taux d'enrôlement à l'enseignement supérieur sur la croissance du produit global au Cameroun pendant la période 1965 - 2002. Néanmoins, ces auteurs recommandent la prudence quant à une généralisation de ce résultat, estimant que la contribution positive de l'enseignement supérieur à la croissance économique est tributaire de l'emploi adéquat des diplômés pour faire valoir les compétences acquises.

Sur le plan théorique, le résultat de la présente étude est conforme à la thèse de Aghion et Cohen (2004) qui ne recommandent l'enseignement supérieur pour la croissance économique que dans les pays proches de la frontière technologique. Il est aussi conforme à la thèse soutenue par Vandenbussche et al. (2006). Ceux-ci n'excluent pas que l'enseignement supérieur puisse avoir un impact négatif pour la croissance économique dans les pays en développement. Cependant, la diversité de résultats des études menées ne permet pas de tirer une conclusion définitive quant au rôle de l'enseignement supérieur dans la croissance de ces pays. On remarque que toutes ces études sont d'ordre quantitatif et il faudra peut être des études qualitatives pour approfondir les connaissances sur l'impact de cet ordre d'enseignement sur l'accroissement de la richesse des pays sous-développés. Pour finir, comme nombre de précédents travaux, il faut reconnaitre que ce travail non plus n'est pas à l'abri du problème récurrent de la qualité des données statistiques. Cela l'est d'autant plus que la plupart des variables utilisées sont une combinaison de données secondaires. Néanmoins, ces données avant publication officielle font l'objet d'enquêtes appropriées et de validation par les experts.

\section{Conclusion et Recommandations}

Ce travail avait pour objectif d'étudier la relation entre l'enseignement supérieur et la croissance économique en Côte d'Ivoire, et d'évaluer éventuellement l'impact du premier sur le second. Pour ce faire, on a utilisé un modèle ARDL et le test de causalité au sens de Granger de Toda et Yamamoto (1995). Le test de cointégration aux bornes de Pesaran et al. (2001) 
a révélé l'existence d'une relation de cointégration entre la croissance du produit global et le stock de capital humain de niveau supérieur. Les résultats des estimations et de l'analyse causale montrent que la croissance économique en Côte d'Ivoire est impactée de façon significative mais négative par l'enseignement supérieur. Ce résultat est le même aussi bien à court terme qu'à long terme. A court terme, un accroissement de $1 \%$ du stock de capital humain de niveau supérieur provoque une baisse de $6,62 \%$ du produit global par travailleur. A long terme, cette baisse sera plutôt de 1,06\%.

Certaines circonstances semblent favoriser et justifier ce fait. On note d'abord la mauvaise qualité du système de formation. Cette piètre qualité peut conduire à une surestimation du stock réel de capital humain, en fournissant à l'économie des travailleurs dont les connaissances ou compétences sont en déçà de ce qu'elles devraient être pour les niveaux d'études affichés. Ensuite, quand la qualité de la formation y est, le diplômé à la fin de sa formation doit obtenir un emploi qui lui permet de valoriser les compétences acquises. S'il se retrouve au chômage ou à un emploi de moindre qualification par rapport à sa formation, son rendement éducatif est alors fortement réduit quand il n'est pas nul. Enfin, un diplômé bien formé peut paradoxalement devenir un obstacle à la bonne marche de l'économie s'il utilise ses connaissances et compétences à des fins de corruption, de détournements, de vols ou d'autres vices. Ainsi, pour inverser l'effet négatif de l'enseignement supérieur sur la croissance, il faudra réformer cet ordre d'enseignement pour améliorer la qualité de la formation des étudiants, améliorer l'employabilité des diplômés de l'enseignement supérieur, faire correspondre le volume et le type de formation aux besoins de l'économie et éviter la sur-éducation. En outre, il faut veiller à développer un environnement institutionnel de qualité et à promouvoir la bonne gouvernance.

\section{References :}

1. Aghion, P., \& Cohen, É. (2004). Éducation et croissance. Paris: La Documentation française. Récupéré sur http://www.caeeco.fr/IMG/pdf/046.pdf

2. Ali, M., Egbetokun, A., \& Memon, M. H. (2018). Human Capital, Social Capabilities and Economic Growth. economies, 6(2). doi:10.3390/economies6010002

3. Armeanu, D. S., Vintila, G., \& Gherghina, S. C. (2018). Empirical Study towards the Drivers of Sustainable Economic Growth in EU-28 Countries. Sustainability, 4(10), 22. doi:10.3390/su10010004

4. Benhabib, J., \& Spiegel, M. M. (1994). The role of human capital in economic development evidence from aggregate cross-country data. Journal of Monetary Economics, Volume 34(2), 143-173. 
5. Bloom, D., Canning, D., Chan, K., \& Luca, D. L. (2014). Higher Education and Economic Growth in Africa. International Journal of African Higher Education, 1(1), pp. 22-57.

6. Boccanfuso, D., Savard, L., \& Savy, B. (2009). Capital humain et croissance : évidences sur données de pays africains. Cahier de recherche (09-15), Université de Sherbrooke. Consulté le 20 mai 2019, sur http://gredi.recherche.usherbrooke.ca/wpapers/GREDI-0915.pdf

7. Colclough, C. (1982). The impact of primary schooling on economic development: a review of the evidence. World Development, Elsevier, 10(3), pp. 167-185.

8. Coulibaly, M. (2013, septembre). Impact des dépenses publiques d'éducation sur la croissance économique en Côte d'Ivoire. European Scientific Journal, 9(25), pp. 443-464.

9. Fonkeng, G. E., \& Ntembe, A. (2009, Mai). Higher education and economic development in Africa: The case of Cameroon. Educational Research and Review, 4(5), pp. 231-246.

10. Gurgand, M. (2000). Sait-on mesurer le rôle économique de l'éducation? Revue française d'économie, 15(2), pp. 121-156.

11. Gyimah-Brempong, K. (2010). Education and Economic Development in Africa. University of South Florida, Department of Economics. Récupéré sur https://www.afdb.org/fileadmin/uploads/afdb/Documents/Knowledge /Session\%20II.2.1_2.\%20Education\%20and\%20Economic\%20Devel opment $\% 20$ in\%20Africa.pdf

12. Gyimah-Brempong, K., Paddison, O., \& Mitiku, W. (2006, avril). Higher Education and Economic Growth in Africa. Journal of Development Studies, 42(3), pp. 509-529.

13. Hair Jr, J. F., Black, W. C., Babin, B. J., \& Anderson, R. E. (2014). Multivariate Data Analysis (éd. Seventh Edition). Harlow: Pearson New International Edition.

14. Hanushek, E. A., \& Woessmann, L. (2016). Knowledge capital, growth, and the East Asian miracle. Science, 351(6271), pp. 344-345.

15. Holland, D., Liadze, I., Rienzo, C., \& Wilkinson, D. (2013). The relationship between graduates and economic growth across countries. Bis Research Paper(110), 72.

16. Jeon, Y. (2007). Total Factor Productivity and Income Distribution: A Critical Review. Working Paper (2007-04), University of Utah, Department of Economics. Récupéré sur http://economics.utah.edu/research/publications/2007_04.pdf

17. Kafimbou, H., Sanon, J., Ouedraogo, A., Zoundi, J., \& Sanon, K. (2011). Enseignement supérieur et développement socio-économique au Burkina Faso. Afr educ dev issues(3), pp. 24-51. 
18. Keho, Y. (2009). Analyse retrospective de l'équité sociale et esquisse d'images à long terme de la société ivoirienne. Abidjan: Cellule d'Analyse de Politiques Economiques du CIRES.

19. Keho, Y. (2012). Le rôle des facteurs institutionnels dans le développement financier et économique des pays de l'uemoa. (BCEAO, éd.) Revue économique et monétaire(12), pp. 11-43.

20. Keller, K. (2006). Investments in primary, secondary, and higher education and the effects on economic growth. Contemporary Economic Policy, 24(1), pp. 18-34.

21. Kocourek, A., \& Nedomlelová, I. (2018). Three levels of education and the economic growth, Applied Economics. Applied Economics, 50(19), pp. 2103-2116. doi:10.1080/00036846.2017.1388910

22. Kohli, U. (2015, novembre). Explaining Total Factor Productivity. University of Geneva.

23. Kotásková, S. K., Procházka, P., Smutka, L., Maitah, M., Kuzmenko, E., Kopecká, M., \& Hönig, V. (2018). The impact of education on economic growth: the case of india. Acta universitatis agriculturae et silviculturae mendelianae brunensis, 66(1), pp. 253-262.

24. Kouton, J. (2018). Education Expenditure and Economic Growth: Some Empirical Evidence from Côte d'Ivoire. Journal of Economics and Sustainable Development, 9(14), pp. 22-34.

25. Kripfganz, S., \& Schneider, D. C. (2019). Response surface regressions for critical value bounds and approximate p-values in equilibrium correction models. Discussion Papers Series (19/01), University of Exeter, Economics Department. Récupéré sur http://people.exeter.ac.uk/RePEc/dpapers/DP1901.pdf

26. Leeuwen, B. v. (2007). Basic data and measurement issues: standard proxy estimates of human capital. Dans B. v. Leeuwen, Human Capital and Economic Growth (pp. 47-75). Récupéré sur http://www.iisg.nl/indonesianeconomy/humancapital/pdf/8chapter3.pdf

27. Mankiw, N. G., Romer, D., \& Weil, D. N. (1992, May). A contribution to the empirics of economic growth. The Quarterly Journal of Economics, pp. 407-437.

28. McNeil, C., \& Silim, A. (2012). Further higher? Tertiary education and growth in the UK's new economy. UCU report. Récupéré sur https://www.ucu.org.uk/media/5278/Further-Higher-TertiaryEducation-and-Growth-in-the-UKs-New-Economy-IPPRUCU-Jun12/pdf/further_higher_report_final.pdf

29. Ministère de l'Enseignement Supérieur et de la Recherche Scientifique. (s.d.). L'Enseignement Supérieur et la Recherche Scientifique en Côte d'Ivoire: Bilan, actions réalisées et perspectives. 
Récupéré

sur http://www.gouv.ci/doc/1351181160Perspectives\%20enseignement\% 20sup.pdf

30. Nowak, A. Z., \& Dahal, G. (2016). The contribution of education to economic growth: evidence from nepal. International Journal of Economic Sciences, V(2), pp. 22-41. doi:10.20472/ES.2016.5.2.002

31. N'Zué, F. F. (2004, Janvier). Le Rôle des Exportations dans le Processus de Croissance Economique de la Côte d'Ivoire: Ses Implications pour des Stratégies de Création d'Emplois Durables. African Development Review, 15(2-3), pp. 199-217. doi:10.1111/j.1467-8268.2003.00071.x

32. Okocha, R. (2015). Capital humain, éducation et croissance économique: Une approche économétrique. Thèse de doctorat SCIENCES ECONOMIQUES, Université Abou Bekr Belkaid, Tlemcen. Récupéré sur http://dspace.univtlemcen.dz/bitstream/112/7231/1/capital-humain-educationcroissance-economique.Doc.pdf

33. Prichett, L. (2001). W here Has All the Education Gone? The World Bank Economic Review, 15(3), pp. 367-391. doi:10.1093/wber/15.3.367

34. Quenum, V. C. (2011). Niveaux d'éducation et croissance économique dans les pays de l'UEMOA. Revue d'Economie Théorique et Appliquée, 1(1), pp. 41-62.

35. Ramanantoanina, P. P. (2017, décembre). La gouvernance de l'enseignement supérieur et de la recherche scientifique en Côte d'Ivoire. L'enseignement supérieur en Côte d'Ivoire, 4(127783). Récupéré sur http://documents.worldbank.org/curated/pt/810341530268496327/pd f/Governance-in-Higher-Education.pdf

36. Solow, R. M. (1956, Février). A Contribution to the Theory of Economic Growth. The Quarterly Journal of Economics, 70(1), pp. 65-94.

37. Taleb, N., \& Bates, S. (2004, septembre). Causalité entre actif boursier et consommation: tentative de réponse à la controverse des effets richesse. Université Paris 13, Centre d'Economie de Paris Nord (UMR 71-15). Récupéré sur https://www.gate.cnrs.fr/t2m2005/Textes\%20des\%20communication s/C67.pdf

38. Urhie, E. (2014). Public Education Expenditure and Economic Growth in Nigeria: A Disaggregated Approach. Journal of Empirical Economics, 3(6), 370-382. 
39. Vandenbussche, J., Aghion, P., \& Meghir, C. (2006). Growth, Distance to Frontier and Composition of Human Capital. J Econ Growth, 11(2), pp. 97-127. 